\title{
Multiscale mass conservative domain decomposition preconditioners for elliptic problems on irregular grids
}

\author{
Andreas Sandvin • Jan Martin Nordbotten • \\ Ivar Aavatsmark
}

Received: 25 May 2010 / Accepted: 26 January 2011 / Published online: 24 February 2011

(C) The Author(s) 2011. This article is published with open access at Springerlink.com

\begin{abstract}
Multiscale methods can in many cases be viewed as special types of domain decomposition preconditioners. The localisation approximations introduced within the multiscale framework are dependent upon both the heterogeneity of the reservoir and the structure of the computational grid. While previous works on multiscale control volume methods have focused on heterogeneous elliptic problems on regular Cartesian grids, we have tested the multiscale control volume formulations on two-dimensional elliptic problems involving heterogeneous media and irregular grid structures. Our study shows that the tangential flow approximation commonly used within multiscale methods is not suited for problems involving rough grids. We present a more robust mass conservative domain decomposition preconditioner for simulating flow in heterogeneous porous media on general grids.
\end{abstract}

Keywords Porous media • Reservoir simulation • Multilevel

\section{Introduction}

The heterogeneities at different scales in porous rocks make reservoir simulations computationally challeng-

\footnotetext{
A. Sandvin $(\bowtie) \cdot$ I. Aavatsmark

Centre for Integrated Petroleum Research,

University of Bergen, 5020, Bergen, Norway

e-mail: andreas.sandvin@cipr.uib.no

J. M. Nordbotten

Department of Mathematics, University of Bergen,

Johannes Brunsgate 12, 5008, Bergen, Norway
}

ing, both with respect to time consumption and accuracy. The rapid variations in fine-scale permeability have big influence on the flow and need to be accounted for in the numerical methods. Various upscaling procedures have been developed to increase the efficiency of the flow calculations (see [12]). These techniques serve to construct coarse-scale flow parameters for the global problem on a coarser scale. However, for flow in complex geological media, it is crucial to solve the transport of fluids on the fine scale (Darcy scale). The idea behind multiscale methods as it was presented in [18] is to capture the fine-scale flow properties within independent local basis functions. After solving for the pressure on the coarse scale, the local basis functions then serve as accurate interpolation functions from the coarse-scale to the fine-scale pressure solution. Since the significant change in saturation often takes place in smaller parts of the global domain, only a few local basis functions need to be recalculated at each time step. Also, since the local basis functions are independent, the calculation of these may be carried out in parallel. Numerical experiments show that the multiscale technique can be efficient for solving multiphase flow problems in heterogeneous porous media $[1,3,20]$.

The accuracy of the multiscale solution will, however, depend on the choice of localisation approximation, i.e. the choice of boundary conditions for the local basis function problems. Since the error in the local solutions is the largest close to the local boundaries, it has been shown in [19] that the error can be greatly reduced by calculating the local basis functions on larger overlapping domains. This corresponds to moving the local boundaries closer to the global boundary which determines the exact flow field. Another approach is to directly incorporate global information into the local 
boundary value problems, by first solving an initial global fine-scale problem [11]. The use of local-global information has also been considered in [10]. While these methods may provide more accurate local solutions, they are in general more computationally expensive, specially in the case of repeated update of the global information, e.g. for changing global boundary conditions. A third approach is to improve the accuracy by means of local iterations on the domain interfaces $[16,28]$. We will consider the latter approach.

It has been showed, in [28], that the multiscale finitevolume (MSFV) method of Jenny et al. [20] can be viewed as a special case of a mass conservative domain decomposition (MCDD) preconditioner, using a tangential flow approximation on the domain interfaces. The fine-scale solution for the MSFV method is expressed as a linear combination of local basis functions, which is equivalent with one iteration using the MCDD preconditioner. In the following, we will refer to the multiscale solution, as the approximation obtained after one iteration with the MCDD preconditioner. The class of MCDD preconditioners offers a general framework for approximating the flow on the interface, in which we can construct a wide range of different multiscale preconditioners with various properties. In this paper, we will focus on four principal properties for the multiscale preconditioner. The preconditioner should:

- Be cheap to construct. In this paper, we consider local sparse approximations to the flow on the boundary, i.e. sparse representations of the local Schur complement systems.

- Be applicable as a multiscale method. For many practical applications, it is computationally too expensive to iterate on the fine-scale solution, and the coarse-scale solution will be applied directly. The preconditioner should give a physically reliable approximation to the fine-scale flow field after only one iteration.

- Possess good convergence properties. Some of the local fine-scale features may be difficult to capture within a coarse-scale system. Thus, we are forced to iterate on the fine-scale residual.

- Be applicable to realistic porous media. Realistic flow problems for reservoir simulation involve irregular grid structures and heterogeneous anisotropic permeability fields. The multiscale approximations induced on the local domain interfaces have to be robust with respect to irregular geometries and fine-scale anisotropies not aligned with the grid.
We will consider the following elliptic problem for two-dimensional flow in porous media,

$-\nabla \cdot(\mathbf{K} \nabla u)=q \quad$ in $\Omega \subset \mathbb{R}^{2}$,

where $\mathbf{K}$ is a symmetric positive definite matrix representing the permeability of the media, $u$ is the potential and $q$ represents the source terms. The permeability is in general a full tensor describing the conductivity of an anisotropic porous medium, and it is the spatial variability of this parameter which represents the key challenge discussed in this paper. By integrating Eq. 1 over an arbitrary control volume $\omega \subset \Omega$ and applying Green's theorem, we obtain the integral equation for conservation of incompressible fluids,

$\int_{\partial \omega} \mathbf{f} \cdot v \mathrm{~d} \sigma=\int_{\omega} q \mathrm{~d} \tau$.

Here, $\mathbf{f}=-\mathbf{K} \nabla u$ represents the Darcy velocity and $v$ is the outward normal vector to $\partial \omega$. Methods based on the discretisation of Eq. 2 is referred to as control volume methods and yield local mass conservation within $\omega$. The resulting discrete system of fine-scale equations, arising from a control volume discretisation, takes the following form:

$A u=b$.

We will assume that the solution $u \in V$, where $V$ is the space of piecewise linear functions on $\Omega=\cup \omega_{i}$. The right-hand side term $b$ represents the integrated sources over $\omega_{i}$ and belongs to the space of piecewise constant functions on $\Omega$. Finally, the fine-scale operator $A \in V$ is a sparse and in general non-symmetric matrix, which contains information about the fine-scale geometry and variability of $\mathbf{K}$.

Previously, multiscale control volume methods have focused on the elliptic problem (see Eq. 1) on regular Cartesian grids. As far as we know, multiscale control volume methods have not been applied to problems involving irregular grid structures. In this paper, we investigate the accuracy, efficiency and robustness of different multiscale control volume approximations when applied to heterogeneous problems on irregular grids. Our study shows that the reduced boundary condition, commonly applied with the MSFV methods [10, $16,20]$, is not robust with respect to perturbations on the fine-scale grid. Even for regular Cartesian fine grids and isotropic permeability tensor, the spatial variability in the fine-scale permeability may produce anisotropies on the coarse scale. The tangential flow on the local 
boundaries is not sufficient to capture these coarsescale anisotropies within the solution. We introduce a new multiscale framework, based on algebraic approximations to the Schur complement for constructing more accurate and robust interface approximations, for multiscale simulations on irregular grids. While this is a pure algebraic technique, it naturally extends to unstructured and multilevel grids.

The paper is organised as follows: In the next section, we give an introduction to the MCDD preconditioners. We show how the multiscale methods can be formulated as stand-alone upscaling methods, or as MCDD preconditioners for an iterative process. In Section 3, we compare the existing localisation approximation used for multiscale control volume methods with an algebraic interface approximation based on probing. A small comparison on the computational cost related to each of the preconditioners is given in Section 4, before testing the robustness and efficiency of the preconditioners for some numerical experiments in Section 5. Finally, we conclude the paper.

\section{MCDD}

In this section, we will consider the framework of non-overlapping domain decomposition methods and introduce the special class of MCDD methods introduced by [28]. Within this framework, we formulate the multiscale control volume methods as MCDD preconditioners.

\subsection{Grids and scales}

We will consider a cell-centred grid on the fine scale, consisting of control volumes $\left\{\omega_{i}, 1 \leq i \leq n\right\}$, such that they form a non-overlapping partition of $\Omega$. Thus,

$\Omega=\bigcup_{i} \omega_{i} ; \quad \omega_{i} \cap \omega_{j}=\emptyset \quad i \neq j$.

A primal coarse grid is then constructed on top of the fine grid, such that each primal coarse-grid cell $\left\{\Omega_{i}, 1 \leq i \leq N\right\}$ is a collection of fine-grid cells and the boundaries of $\Omega_{i}$ coincide with boundaries on the fine grid, as shown in Fig. 1. Moreover, we require that each fine-grid cell is represented in exactly one primal coarse-grid cells. Thus, the primal coarse grid also satisfies a non-overlapping partitioning of $\Omega$. The centremost fine-grid cell within each primal coarse-grid cell is further denoted as the coarse-grid node. Continuing in this manner, we note that we can construct a hierarchy

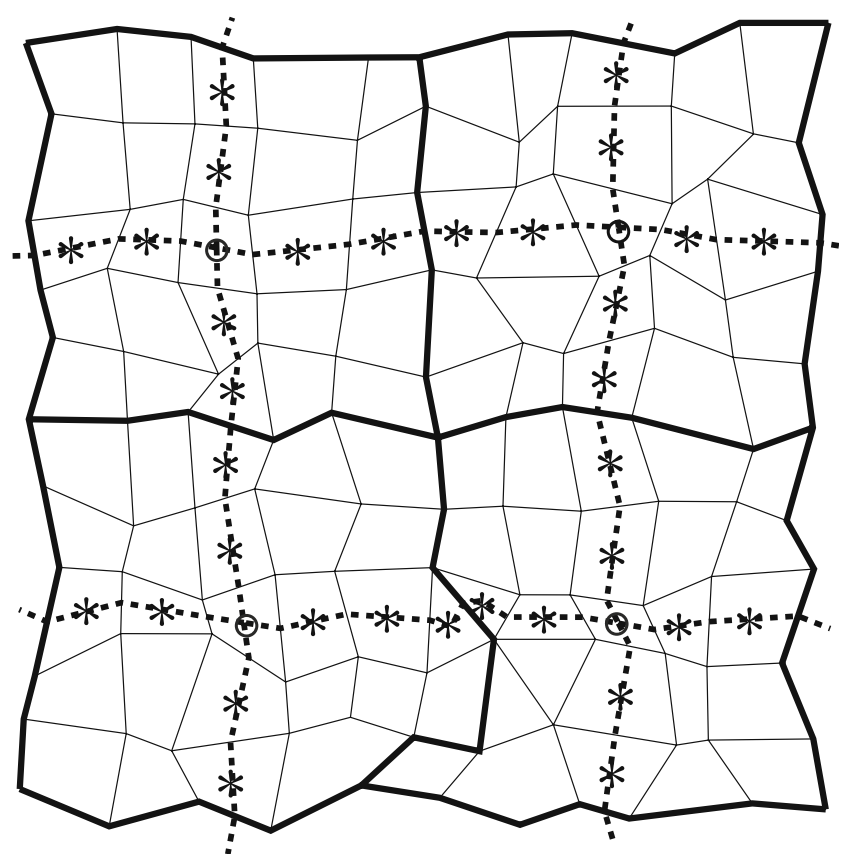

Fig. 1 The multiscale mesh. Here, the bold faces show the primal coarse grid, constructed on top of an underlying fine-scale grid. The dashed lines further indicate the dual coarse grid, on which the circles and stars refer to vertex and edge nodes, respectively

of cell-centred coarse grids. However, for simplicity, we will here restrict our attention to two-scale methods. We consider true multiscale implementations of this framework in [30]. Note also that both the fine and the coarse grid may consist of arbitrarily shaped polygons.

We further introduce a dual coarse grid (indicated by dashed lines in Fig. 1) on which we will solve our local problems. We will refer to the dual coarse-grid cells as domains and denote them by $\Omega_{i}^{\prime}$. The primal coarsegrid nodes of $\Omega$ will then be located at the vertices of $\Omega_{i}^{\prime}$. On each domain $\Omega_{i}^{\prime}$, the degrees of freedom corresponding to the boundary nodes will be denoted by subscript $B$ and those corresponding to internal nodes by subscript $I$. The degrees of freedom related to the boundary unknowns will further be subdivided into those corresponding to vertex nodes and edge nodes, denoted by subscripts $V$ and $E$, respectively.

Note that, while the primal coarse cells $\Omega_{i}$ are collections of cell-centred grid cells, the degrees of freedom on $\Omega_{i}$ are strictly separated from those on $\Omega_{j}$, when $i \neq j$. For the dual coarse grid, the nodes are located on the vertices. Hence, the dual coarse cells $\Omega_{i}^{\prime}$ form a non-overlapping partitioning of $\Omega$ in the classical sense, where the nodes on the dual-cell boundaries may be shared between neighbouring cells. 


\subsection{Mass conservative coarse-scale operator}

For many applications, the multiscale method is applied as an upscaling procedure. At each time step of the simulation, the potential values are solved on the coarse scale, and the fine-scale solution is only reconstructed locally through a linear combination of coarse-scale basis functions. These basis functions are computed initially and seldom recomputed during the simulation. To reconstruct a mass conservative finescale flux field at a given time step, it is crucial that the coarse-scale operator also serves as a discretisation of the mass-conservation principle given in Eq. 2. Thus, as a preprocessing step, we will integrate the fine-scale equations (corresponding to fine-scale control volumes $\left.\omega_{i}\right)$ associated with each primal coarse cell $\Omega_{i}$ into the row of the corresponding coarse node $i$. This will give us mass conservation on the primal coarse grid, represented by the coarse nodes.

We will consider a family of spaces $\left\{V_{i}, 1 \leq i \leq\right.$ $N$ \} corresponding to the primal coarse cells $\Omega_{i}$ and the extension operators $R_{i}^{T}: V_{i} \rightarrow V$, such that $V=$ $\sum_{i=1}^{N} R_{i}^{T} V_{i}$. For each $\Omega_{i}$, we define $M_{i}: V_{i} \rightarrow V_{i}$ as the integration operator adding all rows in $R_{i} A$, corresponding to fine-scale control volume equations on $\Omega_{i}$, into the row of the coarse node $i$. More precisely, we can write the integration operator on matrix form

$M_{i}=I+e_{i V}\left(\underline{1}-e_{i V}\right)^{T}$

where $I$ is the identity matrix, $e_{i V}$ is the unit vector identifying the row of the vertex (coarse) node and 1 is the vector entirely filled with ones. By applying the integration operator $M_{i}$ on the fine-scale system (Eq. 3) restricted to each coarse cell $\Omega_{i}$, we construct a system of equations, which is mass conservative on both scales. We write the MCDD system as

$C u=p$,

where

$C=\sum_{\Omega_{i}}\left(R_{i}\right)^{T} M_{i} R_{i} A$ and $\quad p=\sum_{\Omega_{i}}\left(R_{i}\right)^{T} M_{i} R_{i} b$

The fine-scale operator $C$ belongs to the same space as $A$ but has the additional property of preserving the mass balance on the coarse scale as well as the fine scale. It can be shown that this preprocessing step also acts as a good preconditioner for the fine-scale operator $A$, as it introduces a coarse space [30].

\subsection{Schur complement system}

The idea behind domain decomposition methods is to decouple the global fine-scale problem into independent local boundary value problems. The global fine-scale problem is then solved by iterating on the boundary unknowns of these local problems. To accelerate the iterative process, a global coarse-scale problem is constructed to capture the low-frequency error and to pass information between the local problems.

By grouping the unknowns corresponding to internal nodes in $u_{I}$ and those corresponding to boundary nodes in $u_{B}$, we can reorder the unknowns $u=\left[\begin{array}{ll}u_{I} & u_{B}\end{array}\right]^{T}$, and write Eq. 5 as

$\left[\begin{array}{ll}C_{I I} & C_{I B} \\ C_{B I} & C_{B B}\end{array}\right]\left[\begin{array}{l}u_{I} \\ u_{B}\end{array}\right]=\left[\begin{array}{l}p_{I} \\ p_{B}\end{array}\right]$

All the internal unknowns $u_{I}$ are now decoupled into local domains $\Omega_{i}^{\prime}$, where the matrix $C_{I I}$ has a simple block diagonal structure. Thus, the internal degrees of freedom may be solved locally within each domain $\Omega_{i}^{\prime}$ as

$u_{I}=C_{I I}^{-1}\left(p_{I}-C_{I B} u_{B}\right)$.

Hence, we can eliminate the internal degrees of freedom by substituting Eq. 7 into the second line of Eq. 6 and obtain the reduced Schur complement system

$$
\left(C_{B B}-C_{B I} C_{I I}^{-1} C_{I B}\right) u_{B}=p_{B}-C_{B I} C_{I I}^{-1} p_{I}
$$

The matrix $S=C_{B B}-C_{B I} C_{I I}^{-1} C_{I B}$ is referred to as the Schur complement of $C$, and for simplicity, we also denote $g=p_{B}-C_{B I} C_{I I}^{-1} p_{I}$ as the modified righthand side term. The Schur complement $S$ is related to the space of discrete harmonic functions, where the multiplication of $S$ to a vector $\mathbf{x}$ is equivalent to solve a local Dirichlet problem involving $C_{I I}^{-1}$ on each domain $\Omega_{i}^{\prime}$. The matrix $S$ can be shown to yield better properties w.r.t. the condition number [4]; however, it is expensive to construct. In general, we never explicitly construct the Schur complement matrix $S$; we only do the necessary matrix-vector multiplications involving $S$.

By a similar reordering of the unknowns, $u_{B}=$ $\left[\begin{array}{ll}u_{E} & u_{V}\end{array}\right]^{T}$, we can write the Schur complement system on matrix form

$\left[\begin{array}{ll}S_{E E} & S_{E V} \\ S_{V E} & S_{V V}\end{array}\right]\left[\begin{array}{l}u_{E} \\ u_{V}\end{array}\right]=\left[\begin{array}{l}g_{E} \\ g_{V}\end{array}\right]$

Here, $S_{E E}$ and $S_{E V}$ have a block diagonal structure; however, each block is in general dense. In particular, 
the Schur complement matrix $S_{E E}$ can analytically be written as

$S_{E E}=C_{E E}-C_{E I} C_{I I}^{-1} C_{I E}$.

This equation will be important later, when we discuss the properties of the different preconditioners. We observe that the first term in Eq. 10 contains the local couplings between the neighbouring edge elements, $C_{E E}$. This matrix will be sparse, with a predominantly tridiagonal structure. The second term in Eq. 10 is referred to as the global term, where internal information is interpolated onto the edge nodes. This term couples all the edge nodes together and forms a full matrix.

A direct solution of Eq. 9 is for most applications computationally too expensive, and we seek to construct a preconditioner for the Schur complement problem such that the number of local algebraic operations involving $C_{I I}^{-1}$ is as low as possible. We approximate Eq. 9 by

$\left[\begin{array}{cc}I & \hat{S}_{E E}^{-1} \hat{S}_{E V} \\ S_{V E} & S_{V V}\end{array}\right]\left[\begin{array}{c}u_{E} \\ u_{V}\end{array}\right]=\left[\begin{array}{c}\hat{S}_{E E}^{-1} g_{E} \\ g_{V}\end{array}\right]$

where $\hat{S}_{E E}$ and $\hat{S}_{E V}$ now denote the approximations to $S_{E E}$ and $S_{E V}$, respectively. Note that we have only modified the equations for the edge nodes, where

$u_{E}=\hat{S}_{E E}^{-1}\left(g_{E}-\hat{S}_{E V} u_{V}\right)$.

Hence, the solution $u_{V}$ of Eq. 11 still remains mass conservative on the coarse scale. In fact, any approximation of $u_{E}$ will only affect the accuracy and not the property of mass conservation on the coarse scale. By substituting Eq. 12 into the second line of Eq. 11, we can write a mass conservative system of equations for the solution $u_{V}$ on the coarse scale,

$A_{C} u_{V}=g_{V}-S_{V E} \hat{S}_{E E}^{-1} g_{E}$,

where

$A_{C}=\left[S_{V E} S_{V V}\right]\left[\begin{array}{c}-\hat{S}_{E E}^{-1} \hat{S}_{E V} \\ I\end{array}\right]$.

The coarse-scale operator $A_{C}$ is related to the space of piecewise discrete harmonic functions, where the approximated discrete harmonic extension on $u_{V}$ is determined by the $\hat{S}_{E E}$ and $\hat{S}_{E V}$. For the multiscale methods, the coarse-scale equation (Eq. 13) will be solved directly. The columns of $A_{C}$ contain the coarsescale basis functions, which can be used to recover the fine-scale solution $u_{f}=\left[\begin{array}{lll}u_{I} & u_{E} & u_{V}\end{array}\right]^{T}$.

\section{Interface approximations}

The essential part in the construction of a good MCDD preconditioner, or efficient and accurate multiscale method, is the choice of interface approximation, which in our framework is $\hat{S}_{E B}=\left[\hat{S}_{E E} \hat{S}_{E V}\right]$. For most cases, the approximation error in $\hat{S}_{E E}$ will dominate. In this section, we will primarily focus on different approximations to $S_{E E}$ and use $\hat{S}_{E V}=C_{E V}$. In Section 3.4, we will further discuss approximation techniques for the entire $S_{E B}$ and how this can be related to flow-based upscaling techniques.

Recall from Eq. 10 that $S_{E E}$ consists of two terms, a local term $C_{E E}$ and a global term containing the couplings between edge and internal nodes. We will consider two types of interface approximations: a tangential flow approximation and an interface probing approximation. Both approximations result in lowband matrices, which are fast to invert. We have also applied other interface approximations, like the Toeplitz approximation [5] and the J-operator [6]. While they yield good results for the elliptic problem with constant coefficient on uniform Cartesian grids, they do not perform well for problems involving heterogeneous porous media and non-regular fine grids. Another approach, not considered here, is to directly approximate the second term of Eq. 10 by applying some local preconditioner on $C_{I I}$. This would lead to a more expensive composite preconditioner, where the resulting approximation is not guaranteed to be sparse. For a broader discussion on different interface approximations, see [31].

\subsection{Approximation properties}

Our aim is to construct approximations $\hat{S}_{E E}$ with similar spectral properties as $S_{E E}$ for which the system involving $\hat{S}_{E E}$ is fast to compute. In order to get a physically reliable solution after only one iteration (equivalent to solving the coarse-scale problem), we need to require some properties for the coarse-scale operator $A_{C}$. For single-scale methods, usual requirements for the system matrix $A$ are that they are mass conservative and exactly reproduce constant and linear potential fields. As an example, most control volume methods are constructed precisely to satisfy these criteria.

As shown in Section 2, the MCDD preconditioners are constructed to be mass conservative on both the fine scale and the coarse scale. Furthermore, we will require that $A_{C}$ is exact for constant solutions. For general heterogeneous elliptic problems, this is the only analytical solution that we can identify, which is 
obtained by imposing zero boundary conditions and no internal source terms. For the multiscale methods, this implies that the local basis functions must form a partition of unity, i.e. the sum of the local basis functions is exactly equal to 1 . For the MCDD preconditioners, we require that

$\hat{S}_{E E} \mathbf{1}=S_{E E} \mathbf{1}$,

which ensures that the approximation $\hat{S}_{E E}$ is exact for constant solutions. In the case of $\mathbf{K}$-orthogonal fine grids, i.e. grids which are aligned with the principal directions of the permeability tensor, there are no couplings between vertex and internal nodes. Thus, $S_{E V}=$ $C_{E V}$ and property 15 is a sufficient criterion for the resulting preconditioner to preserve constant solutions locally. However, for general grids, property 15 is not sufficient and we need to require that

$\hat{S}_{E B} \mathbf{1}=S_{E B} \mathbf{1}$.

The importance of capturing the constant solution has also been emphasized in domain decomposition, where it corresponds to capturing the null space of the local Schur complement matrices [24]. This is one of the important properties for the coarse space, which is needed to construct scalable two-level preconditioners with good convergence rates. Another important property for the coarse space, which is necessary to construct a robust preconditioner, is often referred to as the bounded energy condition [25] and is directly related to the capturing of sub-scale variations in the coefficients of $\mathbf{K}$ [15].

The motivation behind the multiscale methods is precisely to capture these local sub-scale variations within coarse-scale basis functions (i.e. within the coarse space of the corresponding operator $A_{c}$ ), by solving local PDEs on $\Omega_{i}^{\prime}$ with pre-described boundary conditions. By solving extended local problems, e.g. by an oversampling procedure $[9,18]$, the local PDE-based fields on $\Omega_{i}^{\prime}$ are made less sensitive to the boundary approximations, and the sub-scale information along $\partial \Omega_{i}^{\prime}$ can also be well captured.

In Section 3.4, we will show how the interface probing technique can be applied to capture a few PDE-based fields governed by pre-defined boundary conditions on extended local domains. The difference from oversampling and global methods is that the local solutions are applied to approximate the Schur complement $S_{E B}$, rather than the basis functions.

\subsection{Tangential component approximation}

A frequently applied approximation for the multiscale methods, the reduced boundary condition (see [10, 11,
$18,20])$, is to approximate the tangential flow along each local boundary. For the elliptic problem on regular Cartesian grids and with isotropic medium, the tangential flow along the boundary is found by discretising the elliptic equation (Eq. 1) directly along each local edge. As far as we know, the reduced boundary condition has only been applied to regular Cartesian grids, and it is not clear how to extend it to non-K-orthogonal grids. In order to test the reduced boundary condition within the MCDD framework, we discretise the elliptic equation (Eq. 1) along the local boundaries by a two-point flux approximation method. We denote the tridiagonal approximation resulting from the reduced boundary condition by $S_{E E}^{\mathrm{RBC}}$. The preconditioner is denoted MCDD-RBC.

An equivalent approximation for regular Cartesian grids is the tangential component approximation, discussed in [28, 31]. This is an approximation to the first term of Eq. 10. Essentially, the tangential component (TC) approximation splits $C_{E E}=C_{E E}^{T}+C_{E E}^{N}$, where $C_{E E}^{N}$ is a diagonal matrix containing the contribution to normal flow arising from the coupling between edge and internal nodes. By neglecting the flow normal to the local boundaries, the tangential component approximation is defined as

$S_{E E}^{\mathrm{TC}}=C_{E E}^{T}$.

The matrix $S_{E E}^{\mathrm{TC}}$ is tridiagonal when the edge nodes on the dual coarse grid have a natural numbering along each individual interface, and the expression is valid for general grids. We denote the resulting preconditioner, MCDD-TC. In the case of $\mathbf{K}$-orthogonal fine grid, $S_{E E}^{\mathrm{TC}}=S_{E E}^{\mathrm{RBC}}$.

\subsection{Probing technique}

The interface probing technique (see [7] and references therein) represents a more general approach for approximating the flow on the boundary. The aim is to approximate the Schur complement matrix $S_{E E}$ by a low-bandwidth matrix $\hat{S}_{E E}$, such that

$\hat{S}_{E E} \mathbf{v}^{i}=S_{E E} \mathbf{v}^{i}=\mathbf{w}^{i}$,

for some linearly independent probing vectors $\mathbf{v}^{i}$. The method was motivated by the observation that the Schur complement matrix often has a banded structure, where the Schur complement elements decay rapidly away from the diagonal. In fact, it has been shown by Golub [14] that $\left|S_{i j}\right|=O\left(|i-j|^{-2}\right)$, for $i \neq j$. The 
probing vectors suggested in [7] for approximating the $n$-diagonal matrix $S_{E E}^{n P}$ are

$$
\mathbf{v}^{i}=\sum_{j=i \bmod (n)} \mathbf{e}_{j}
$$

In the case of tridiagonal probing, the probing vectors are $\mathbf{v}^{1}=\left[\begin{array}{lllllll}1 & 0 & 0 & 1 & 0 & 0 & \cdots\end{array}\right]^{T}, \mathbf{v}^{2}=\left[\begin{array}{lllllll}0 & 1 & 0 & 0 & 1 & 0 & \cdots\end{array}\right]^{T}$ and $\mathbf{v}^{3}=\left[\begin{array}{lllllll}0 & 0 & 1 & 0 & 0 & 1 & \cdots\end{array}\right]^{T}$. While these probing vectors are linearly independent, Eq. 18 can be shown to yield a unique tridiagonal approximation $\hat{S}_{E E}$. Note also that Eq. 18 does not require the explicit formulation of the full matrix $S_{E E}$, which would be expensive; only those dimensions associated with the probing vectors $\mathbf{v}^{i}$ need to be calculated. In the case of three probing vectors, three local Dirichlet problems needs to be solved.

The interface probing technique can also be thought of as an efficient way of incorporating fine-scale properties into the coarse-scale operator. Indeed, since the sum of the probing vectors represented by Eq. 19 equals $\mathbf{1}$, property 15 is naturally satisfied. We will denote the probing vectors of Eq. 19 as oscillating probing vectors because of their form and since they are designed to capture the fine-scale oscillations of the residual. We refer to MCDD-3P and MCDD-5P as the MCDD preconditioners, using $S_{E E}^{n P}$ with $n=3$ and 5, respectively.

\subsection{Solution-based probing vectors}

Originally, the interface probing technique was applied together with oscillating probing vectors, on the form given by Eq. 19, to approximate the diagonal structure of $S_{E E}$. For heterogeneous and anisotropic problems, there might be strong non-local couplings between boundary nodes, in which case the local Schur complement matrix does not have a diagonal structure. The oscillating probing vectors are designed to capture the fine-scale oscillations of the residual and therefore yield robust and good convergence properties for the iterative process. However, these probing vectors are not able to capture the correct features of the finescale solution after only one iteration. Motivated by standard flow-based upscaling strategies, see, e.g. [12], solution-based probing vectors are introduced to capture the underlying fine-scale variations within each local domain $\Omega_{i}^{\prime}$. Numerical experiments show that these probing vectors can be used to construct preconditioners with better approximation properties for the first iteration [30].

In order to guarantee that the multiscale preconditioners exactly reproduce constant solutions for gen- eral grids, we need to consider the probing of $S_{E B}=$ $\left[S_{E E} S_{E V}\right]$ (see Eq. 16),

$\hat{S}_{E B} \mathbf{v}^{i}=S_{E B} \mathbf{v}^{i}=\mathbf{w}^{i}$.

Within an iterative solution process, we never construct the Schur complement matrix $S_{E B}$ explicitly, nor do we construct the inverse of this matrix. We only apply the $S_{E B}$ to some residual vectors $\mathbf{r}_{B}$ on the boundary. The multiplication of $S_{E B}$ with a probing vector $\mathbf{v}$ can be regarded as solving a local Dirichlet problem with boundary values $\mathbf{v}$. Thus, the accuracy of the preconditioner will depend on how well the probing vectors $\mathbf{v}^{i}$ are able to capture the correct fine-scale variations on the boundary. For example, the choice $\mathbf{v}=\mathbf{1}$ will guarantee that the method preserves constant solutions locally, for general grids.

We compute the solution-based probing vectors $\mathbf{v}_{S B}^{i}$ on $\Omega_{i}^{\prime}$ by solving local flow problems on $\Sigma_{i} \supset \Omega_{i}^{\prime}$. The solution-based probing vectors $\mathbf{v}_{S B}^{i}$ are then defined as the restriction of the local solutions to the local boundary (see Fig. 2). It follows from Eq. 20 that the approximation $\hat{S}_{E B}$ will be exact for those particular boundary value problems defined by $\mathbf{v}_{S B}^{i}$. In this manner, the interface probing approximation can be constructed to be accurate for certain pre-defined boundary value problems.

The resulting multiscale preconditioner shares many similarities with the oversampling technique, applied in $[9,11,13,19]$. In both strategies, a local elliptic problem is solved on an overlapping domain $\Sigma_{i} \supset \Omega_{i}^{\prime}$, in order to reduce the approximation error introduced by the local boundary conditions. The difference of this strategy compared with other extended local or global multiscale methods is that we approximate the Schur complement, not the local basis functions. Thus, our multiscale method is formulated as a Schur complement preconditioner, which makes it a convergent method. Together with solution-based probing vectors, our preconditioner will be accurate for certain righthand sides, in which case it has the additional property of an upscaling method. Results from Sandvin (to be submitted for publication) show that the proposed method is comparable in accuracy to multiscale control volume methods using oversampling.

In order to construct robust approximations to $S_{E B}$, we must require that the probing vectors are linearly independent and that the $\mathbf{1}$-vector is represented through one or a sum of the probing vectors. A combination of solution-based and oscillating probing vectors is in our experience a good choice. In the original framework of the probing technique, a typical choice of $\hat{S}_{E E}$ would be a three-diagonal matrix, constructed from three 
Fig. 2 Local domain $\Omega_{i}^{\prime}$

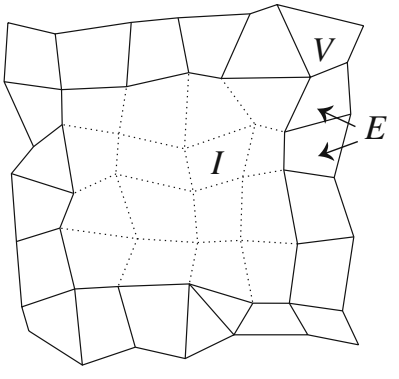

probing vectors, with the same sparsity pattern as the local discretisation $C_{E E}$. Thus, each edge segment connecting two vertex nodes (see Fig. 2) is decoupled, and the edge approximation $\hat{S}_{E E}$ can be inverted locally on the individual edge segments. The approximation $\hat{S}_{E B}$ is constructed in the same manner, from Eq. 20, filling only the diagonal elements and the element neighbours corresponding to the largest couplings in $C_{E B}$. While this interface approximation is only based on neighbour connections, it is a purely algebraic construction and independent on the underlying geometry. We denote the interface approximation $S_{E B}^{N}$ and the corresponding MCDD preconditioner MCDD-N.

The construction of local approximations $\hat{S}_{E B}$, in the case of solution-based probing vectors, can be sensitive w.r.t. the requirement of linear independent probing vectors. To make the proposed MCDD-N preconditioner more robust, it is convenient to add a fourth probing vector and consequently a fourth non-local coupling. As a fourth coupling in $S_{E B}$, we choose an average value, representing the contribution from all local vertex nodes. Thus, the $\hat{S}_{E V}$ will be a full matrix, while the $\hat{S}_{E E}$ retain its local structure and can be inverted locally.

\subsection{Inclusion of global information}

For the application of multiphase flow, the elliptic problem needs to be solved repeatedly in time for slightly varying tensor coefficients $K_{i j}$. In this case, we may afford to spend some extra computational work initially, to construct an accurate multiscale method for the time-dependent problem. In $[10,11]$, they propose a more accurate multiscale method by incorporating information from a global fine-scale solution into the framework of the reduced boundary condition. However, the reduced boundary condition may not be able to capture the local variations of the global information correctly.

The interface probing technique represents a consistent framework for including fine-scale information into operators on coarser scales. In fact, if one of the probing vectors is chosen as the exact fine-scale solution restricted to the boundary, we have not done any approximations on the solution, and the fine-scale solution is solved exactly in one iteration.

\subsection{Comparison of the two interface approximations}

By applying the multiscale method as a preconditioner for a domain decomposition method, we have formulated each local problem as a Schur complement problem. In this framework, the reduced boundary condition, analogous to the tangential component approximation for $\mathbf{K}$-orthogonal grids, is a purely local approximation, i.e. an approximation to the first term in Eq. 10. The algebraic approximation, resulting from the interface probing technique, can be regarded as a global approximation to the Schur complement matrix, where the resulting matrix has a local structure. Let us consider the tridiagonal probing technique described in Section 3.3. From the Eqs. 10 and 18, we have that

$$
\begin{aligned}
S_{E E} \mathbf{v}^{i} & =\left\{C_{E E}^{T}+\left(C_{E E}^{N}-C_{E I} C_{I I}^{-1} C_{I E}\right)\right\} \mathbf{v}^{i} \\
& =\left(\mathbf{w}^{T}\right)^{i}+\left(\mathbf{w}^{N}\right)^{i}
\end{aligned}
$$

where we have split $C_{E E}$ into a tangential component $C_{E E}^{T}$ and a normal component $C_{E E}^{N}$. While the tangential component $C_{E E}^{T}$ has a tridiagonal structure, it is exactly represented by the 3 probing vectors $\mathbf{v}^{i}$ given by Eq. 19, resulting in the tangential component approximation. Thus,

$S_{E E}^{3 P}=S_{E E}^{\mathrm{TC}}+\hat{S}_{E E}^{N}$,

where the second term is an approximation to the flux, accounting for the normal flow on the boundary. Since

$$
\sum_{i} \mathbf{v}^{i}=1
$$

we observe from Eq. 18 that property 15 is satisfied for all interface probing approximations $S_{E E}^{n P}$. However, from Eq. 21, we observe that the tangential component approximation only preserves constant solutions locally whenever

$\left(C_{E E}^{N}-C_{E I} C_{I I}^{-1} C_{I E}\right) \mathbf{1}=0$.

Here, $C_{E E}^{N}$ is a diagonal matrix corresponding to the normal flow, where $C_{E E}^{N} \mathbf{1}=-C_{E I} \mathbf{1}$. Hence,

$C_{E I}\left(\mathbf{1}+\mathbf{y}_{\mathbf{I}}\right)=0$ 
where $\mathbf{y}_{\mathbf{I}}$ can be calculated from

$C_{I I} \mathbf{y}_{\mathbf{I}}=C_{I E} \mathbf{1}$

In case of K-orthogonal grid along the domain boundaries, there are no contributions to the flow between vertex and internal nodes $\left(C_{I V}=\mathbf{0}\right)$, and it follows that relation 26 is equivalent to a Dirichlet problem with constant boundary values -1 ,

$\left[\begin{array}{cc}C_{I I} & C_{I B} \\ 0 & I\end{array}\right]\left[\begin{array}{l}\mathbf{y}_{\mathbf{I}} \\ \mathbf{y}_{\mathbf{B}}\end{array}\right]=\left[\begin{array}{c}0 \\ -1\end{array}\right]$

Thus, $\mathbf{y}_{\mathbf{I}}=-\mathbf{1}$ and Eq. 25 is satisfied. However, for general grid structures, $C_{I V} \neq \mathbf{0}$, and the tangential component approximation does not satisfy property 15 . By probing the $S_{E B}$ (see Eq. 20), the interface probing technique also satisfies property 16 and exactly reproduces constant potential solutions locally, for general grid. Moreover, by choosing solutionbased probing vectors, the preconditioner may be constructed to be exact for any predefined characterisation of the solution.

\section{Computational efficiency aspects}

The main objective of the multiscale methods is efficiency, and the approximations obtained in Section 3 will often be used directly to solve the reduced coarsescale equation (Eq. 13). In the framework of domain decomposition, we may also use these approximative systems as coarse-scale preconditioners for solving the elliptic fine-scale problem iteratively. In this section, we study the computational work related to these preconditioners.

\subsection{Cost of applying the preconditioners}

The MCDD preconditioners discussed in this paper are non-overlapping and residual free on the internal degrees of freedom, meaning that the unknowns corresponding to internal nodes are solved exactly. For an iterative solution process, the internal degrees of freedom need only be resolved once. The degrees of freedom related to the edge nodes are eliminated by the different choices of interface approximations $\hat{S}_{E B}$. The linear system related to $\hat{S}_{E B}$ is assumed to be fast to compute. Thus, the main degrees of freedom are proportional to the number of coarse cells (vertex nodes), and the efficiency of the iterative procedure will be measured due to the number of fine-scale solves needed to solve the coarse-scale equation (Eq. 13).
The major computational cost involves $C_{I I}^{-1}$ applied to vectors $\mathbf{x}_{i}$. These operations require solving the local fine-scale problem

$C_{I I} \mathbf{y}_{i}=\mathbf{x}_{i}$.

The solution vectors $\mathbf{y}_{i}$ can be stored and reused in an iterative process. On each local domain $\Omega_{i}^{\prime}$, we have four degrees of freedom, one related to each vertex node. Thus, the left-hand side of the coarsescale equation (Eq. 13) requires solving four fine-scale problems of the form (Eq. 28). The solutions $\mathbf{y}_{i}$ may be stored as coarse-scale basis functions. For each new iteration, we only need to solve one fine-scale problem corresponding to the new right-hand side term (residual). In practice, we will only update the residual locally on the boundary, in regions where the residual is large.

If the purpose is to solve the coarse-scale equation (Eq. 13) only, the right-hand side term $p_{I}$ corresponding to internal source terms on the fine scale has to be interpolated onto the vertex nodes. This requires solving two additional local fine-scale problems on each local domain where $p_{I} \neq 0$. In the case of $\mathbf{K}$-orthogonal fine grid, $S_{V E}=C_{V E}$, and we only need to solve one additional local fine-scale problem.

\subsection{Cost of constructing the interface approximations}

The accuracy and efficiency of the different MCDD preconditioners lie in the construction of $\hat{S}_{E B}$. In this study, we have only considered low-band approximations $\hat{S}_{E B}$, for which the system $\hat{S}_{E B} \mathbf{y}_{i}=\mathbf{x}_{i}$ is fast to compute. There is, however, an initial cost related to the construction of some of these approximations. For the tangential component approximation and the reduced boundary condition approximation, $\hat{S}_{E B}$ can be constructed directly from the global system matrix $C$ of the fine-scale equation (Eq. 5). The probing technique (see Eq. 18) requires solving one fine-scale problem per probing vector, i.e. 3 and 5 fine-scale problems for the construction of $S_{E B}^{3 P}$ and $S_{E B}^{5 P}$, respectively. The same applies to $S_{E B}^{N}$. However, the fine-scale problems

Table 1 Number of fine-scale solves related to the different MCDD preconditioners

\begin{tabular}{lccccl}
\hline & $S_{E B}^{\mathrm{TC}}$ & $S_{E B}^{\mathrm{RBC}}$ & $S_{E B}^{3 P}$ & $S_{E B}^{5 P}$ & $S_{E B}^{N}$ \\
\hline Interface approximation & 0 & 0 & 3 & 5 & $3-5^{\mathrm{a}}$ \\
Multiscale method & 4 & 4 & 7 & 9 & $7-9^{\mathrm{a}}$ \\
For each new iteration & +1 & +1 & +1 & +1 & +1 \\
\hline
\end{tabular}

${ }^{\text {a }}$ The fine-scale problems related to the solution-based probing vectors can be larger, depending on the size of the overlapping region, on which they are computed 
related to the construction of the solution-based probing vectors might be larger, depending on the size of the overlapping region on which they are computed.

All interface approximations can be reused in an iterative process. An overview of the cost related to each of the preconditioners is summarized in Table 1.

\section{Numerical experiments and results}

The MCDD preconditioners described in the previous subsections are tested for several test problems, involving both irregular grids and heterogeneous permeability fields. The different preconditioners will be compared with respect to the accuracy of the first iteration (equivalent to upscaling) and the number of fine-scale solves to obtain a certain tolerance value for the error (fine-scale solver). While there is an initial cost related to the construction of the preconditioners discussed in Section 4, we choose to compare the preconditioners with respect to the number of local fine-scale solves rather than the number of iterations. The global fine-scale solution will here serve as the reference solution for the approximated solutions.

For the iterative scheme, we apply the preconditioned generalized minimal residual (GMRES) [29], where we compare the efficiency of the different MCDD preconditioners based on the tangential component approximation (MCDD-TC), the reduced boundary condition (MCDD-RBC), the tridiagonal and pentadiagonal probing technique (MCDD-3P and MCDD$5 \mathrm{P})$ and the interface probing technique based on neighbour connections (MCDD-N $(n)$ ). Here, $n$ is the number of overlapping sub-domains, used to compute the solution-based probing vectors. For the numerical results of MCDD-N, we have considered two solutionbased and two oscillatory (see Eq. 19) probing vectors. The two solution-based vectors are constructed, so to capture the principal flow in the horizontal and vertical direction, respectively. As boundary conditions for the overlapping domain, we have applied a unit pressure drop in one direction and no-flow conditions in the other. The MCDD preconditioners are also compared to the unpreconditioned GMRES method, referred to as MCDD-unprec. For large linear systems, the GMRES algorithm requires large information storage, and a restarted version of GMRES may improve the efficiency of the algorithm. For our numerical experiments, we do not consider restarts.

Monotonicity For the fine-scale discretisation, we consider the multipoint flux approximation (MPFA) method as described in [2]. More precisely, we have applied the MPFA O-method, which guarantees continuity of flux over each interface and continuity in pressure at the mid-point of each interface. For general quadrilateral grids, the discretisation will lead to a ninepoint stencil on the fine scale. For K-orthogonal fine grid, the method will reduce to a five-point scheme similar to the two-point flux approximation method. In any case, the coarse-scale operators resulting from the various MCDD preconditioners will in general be nine-point stencils. Common for all these stencils is that they do not guarantee monotone methods [27] and may produce non-physical oscillations on the coarse scale for certain anisotropies on the coarse scale. This may again lead to incorrect flow fields on the fine scale. The solution can, however, be improved through local iterations on the fine scale. Monotonicity of the coarsescale operator for the MSFV method has been studied in [17]. A compact coarse-scale operator with improved monotonicity properties was proposed, which reduces to a seven-point stencil in the limit of homogeneous permeability. However, the compact operator did not lead to improved robustness for the MCDD preconditioners when solving heterogeneous flow problems on irregular grids.

Error norms For the accuracy of the multiscale solution in potential and flux after one iteration, we

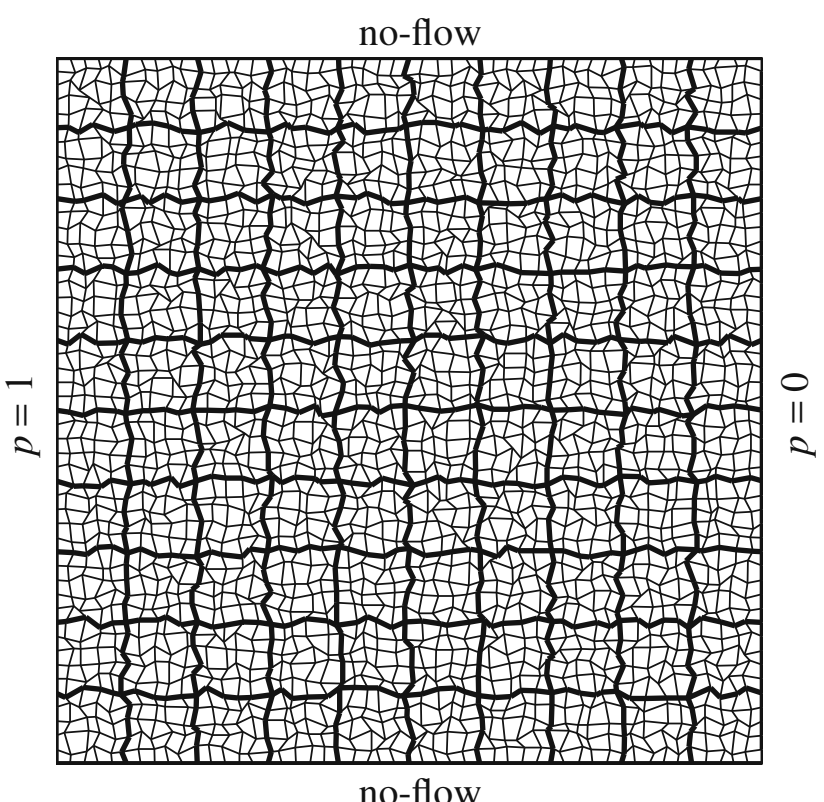

Fig. 3 Computational mesh. The figure shows a $50 \times 50$ irregular Cartesian grid on the fine scale. The degree of perturbation for this grid is $\epsilon=0.4$. The bold faces show the coarse-scale grid, where we have applied a uniform coarsening of $5 \times 5$ grid cells 
consider the error in $L^{\infty}$-norm, as it relates to monotonicity. We denote

$\delta_{u}=\frac{\left\|u^{1}-u^{\infty}\right\|_{\infty}}{\max \left(u^{\infty}\right)-\min \left(u^{\infty}\right)}$,

$\delta_{f}=\frac{\left\|f^{1}-f^{\infty}\right\|_{\infty}}{\max \left(f^{\infty}\right)-\min \left(f^{\infty}\right)}$,

as the multiscale errors for the potential $u$ and the flux $f$, respectively. In Eqs. 29 and 30, $u^{1}$ and $f^{1}$ represent the approximated multiscale solutions (solutions after one iteration), while $u^{\infty}$ and $f^{\infty}$ represent the converged fine-scale solutions for potential and flux. The reconstruction of a mass conservative flux on the fine scale is performed by a post-processing step similar to the MSFV method. The errors within the GMRES algorithm is evaluated in the $L^{2}$-norm.

Grids The MCDD preconditioners are tested on irregular rough grids. These grids are generated by ran-
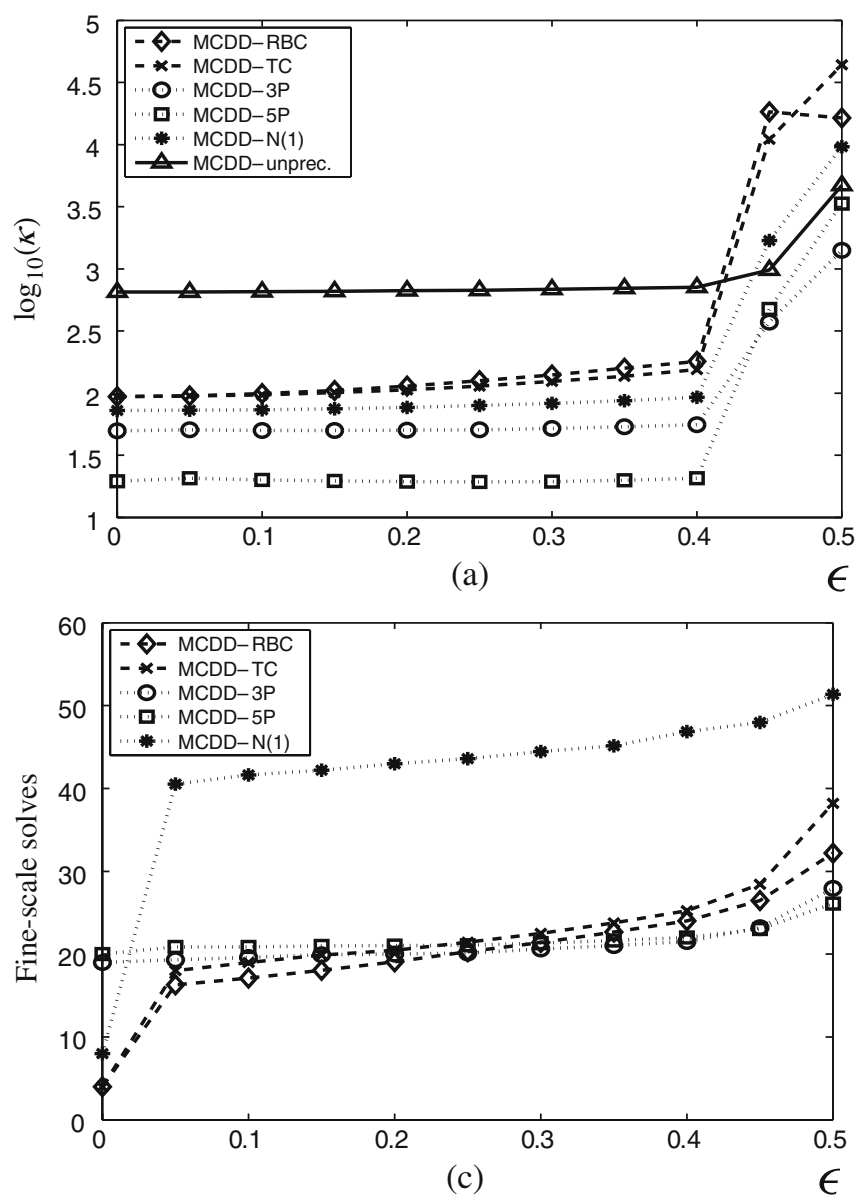

Fig. 4 A comparison of the different MCDD preconditioners for the elliptic problem with constant coefficient. Here, the horizontal axis represent the degree of roughness for the irregular grid. a The spectral condition number and $\mathbf{c}$ the number of fine-scale dom perturbations on the uniform Cartesian grid. If we consider $\mathbf{x}$ to be the coordinates of the initial uniform grid, irregular fine grids are generated by

$\mathbf{x}_{\epsilon}=\mathbf{x}+\epsilon \mathbf{r} h$,

where $h$ is the fine-grid cell size of the initial uniform grid, $\mathbf{r} \in[-1,1]$ is a random variable and $\epsilon \in[0,0.5]$ is the degree of perturbation. Figure 3 shows one example of a simulation grid, where $\epsilon=0.4$.

\subsection{Uniform flow on rough grids}

We first consider the elliptic problem (Eq. 1), where we neglect the source terms $(q=0)$ and apply a homogeneous and isotropic permeability tensor with diagonal elements, $k=1$. We use no-flow boundary conditions on the top and bottom boundary and a unit pressure drop in the horizontal direction, as shown in Fig. 3. From the choice of boundary conditions, the flow on
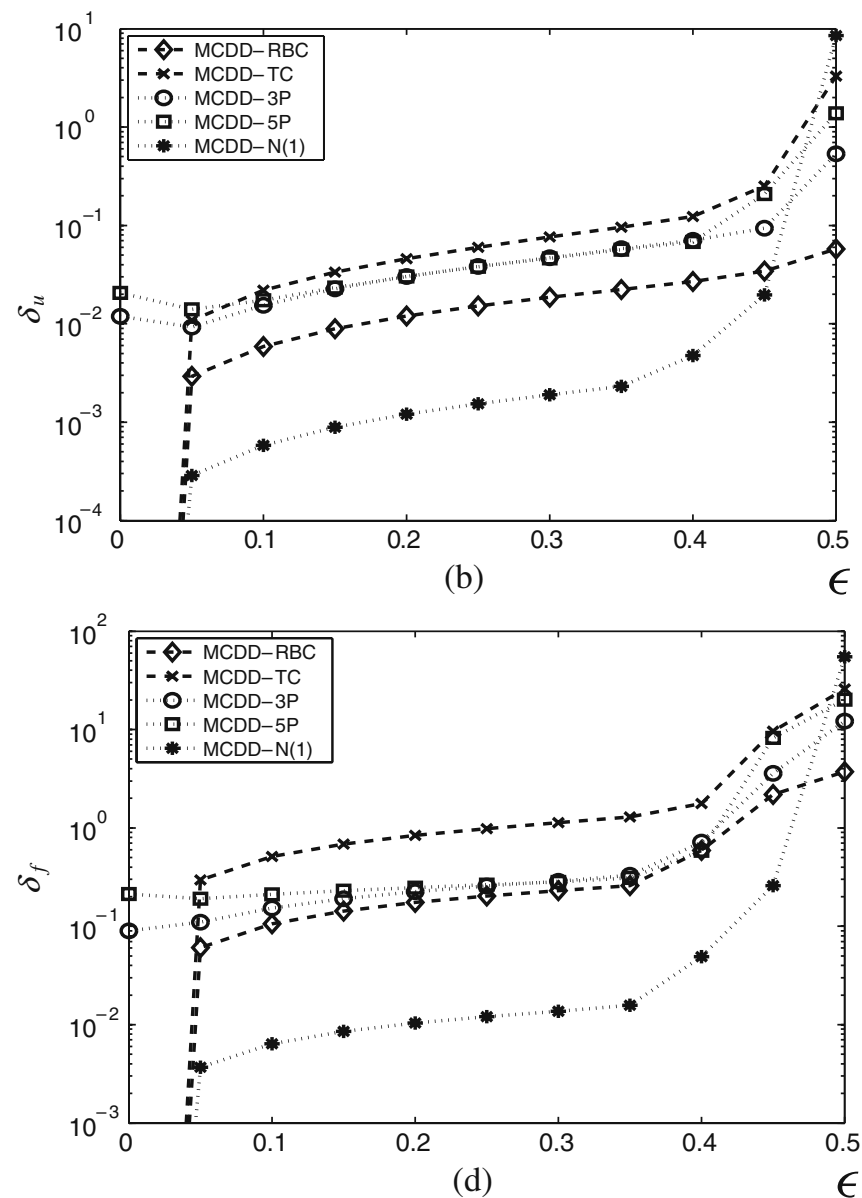

solves to meet a tolerance of $10^{-8}$ for the different preconditioners. In $\mathbf{b}$ and $\mathbf{d}$, we plot the multiscale error, measured in $L^{\infty}$. norm, obtained after one iteration with GMRES. All results are means of 50 realisations of random generated rough grids 
the fine scale will be uniform. We will solve the homogeneous problem on irregular grids, to study the grid dependence of the different MCDD preconditioners. From the construction of control volume methods for elliptic problems, the expression for the flux depends on both geometry and the permeability tensor $\mathbf{K}$, where perturbations on the grid will have similar effects as perturbing $\mathbf{K}$.

Figure 4a shows the comparison of the spectral condition number for the fine-scale system, when applying the different MCDD preconditioners. We observe that the preconditioners based on oscillatory probing vectors (MCDD-3P and MCDD-5P) result in significantly lower condition numbers than the preconditioners based on harmonic (MCDD-RBC) and solution-based vectors (MCDD-N). We also observe that the MCDD$3 \mathrm{P}$ and MCDD-5P preconditioners are more robust with respect to perturbations on the fine-scale grid, for perturbations up to about $40 \%$ of the fine-grid cell size. This indicates that the interface approximations, commonly used for multiscale and upscaling methods, are not as well suited as multiscale preconditioners for the fine-scale problem. For perturbations above $40 \%$ of the fine-grid cell size, none of the low-band approximations for the Schur complement discussed in this paper are robust. Such rough grids will include nonconvex and highly distorted grid cells (see Fig. 3).

In Fig. 4b, we show the number of fine-scale solves, required to solve the homogeneous fine-scale problem. For Cartesian grid $(\epsilon=0)$, the local tangential flow approximations used in MCDD-RBC and MCDD-TC reduce to the exact linear boundary conditions, and the problem is solved exactly in one iteration. However, for perturbed grids $(\epsilon>0)$, the interface probing approximation used in (MCDD-3P and MCDD-5P) are more robust, and the preconditioners need about the same amount of computational work. The MCDD-N preconditioner is designed as an upscaling technique and is not suited as a preconditioner for the iterative scheme. Note also that the MCDD-TC preconditioner, which is a consistent approximation to the tangential flow along perturbed local boundaries, is less accurate than the inconsistent two-point flux approximation used in MCDD-RBC.

In some cases, we cannot afford to iterate on the fine-scale solution, and we would like to reconstruct a fine-scale approximation from the solution of the coarse-scale problem. This is equivalent with one iteration on the fine-scale solution. Figure $4 b, d$ show the accuracy of the multiscale solutions, obtained after one iteration on the fine-scale solution. While the oscillating probing vectors used in MCDD-3P and MCDD-5P result in more robust approximations for the iterative process, they are not as well suited for upscaling. The reduced boundary approximation (MCDD-RBC) has previously shown to be accurate for problems involving regular Cartesian grids; however, it is not as accurate for problems involving irregular grid. By introducing solution-based probing vectors in MCDD-N, we are able to obtain much more accurate approximations to the fine-scale solution at first iteration, for problems involving irregular grids.

\subsection{Heterogeneous problems on rough grids}

Next, we consider elliptic problems with variable coefficients $k(\mathbf{x})$. We consider isotropic, log-normal permeability on the fine scale, as shown in Fig. 5. Local sub-scale heterogeneities may introduce strong nonlocal couplings between boundary unknowns on the local sub-domains, as discussed in Section 3.4, which are more difficult to capture within local interface approximations. From Fig. 6, we see that the MCDD preconditioners resulting from the interface probing technique are able to better capture these non-local couplings and are more robust with respect to fine-scale perturbations on the fine grid. The tangential component approximation is not able to capture the correct flow normal to these local boundaries and has a larger dependency on the degree of perturbation $\epsilon$. The multiscale methods have shown to have difficulties with capturing large anisotropies in the fine-scale flow field, especially for

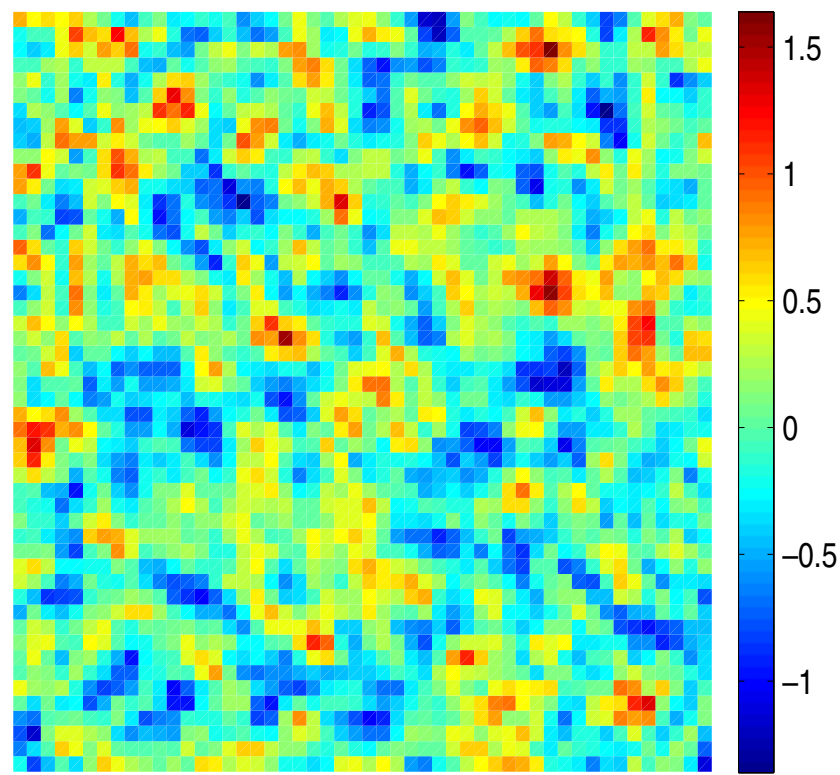

Fig. 5 A generated log-normal permeability field, with standard deviation of 0.5 and a correlation length of 3 fine-grid cells in both $x$ - and $y$-direction. The figure shows the base 10 logarithm of the permeability 


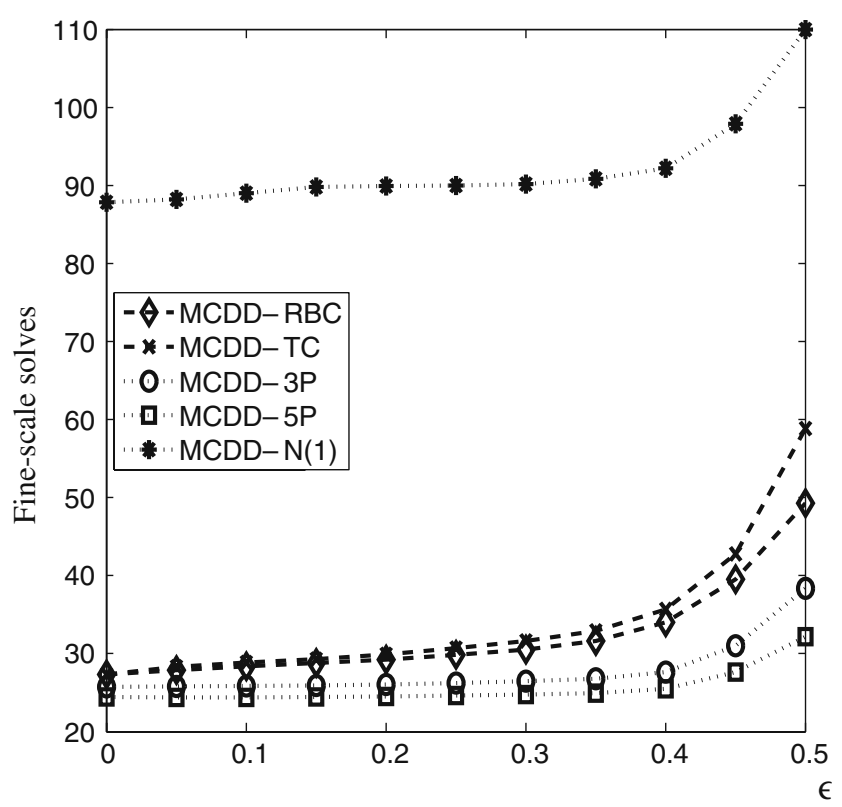

Fig. 6 The number of fine-scale solves needed to reach a tolerance of $10^{-8}$ when solving the elliptic problem for heterogeneous log-normal permeability when applying different MCDD preconditioners. The horizontal axis represents the degree of roughness for the perturbed grid. All results are mean values from 20 realisations of permeability fields and 20 realisations of randomly generated rough grids

problems with diagonal channels going through corner cells of the local sub-domains [22]. This is because the coupling, $C_{V I}$, between vertex and internal nodes, is

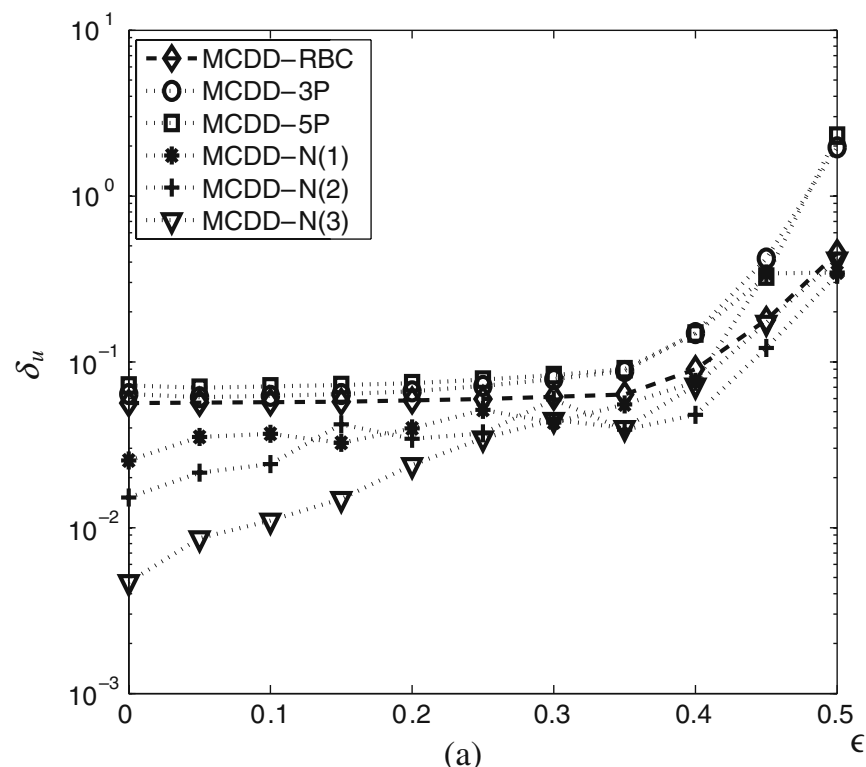

(a)

Fig. 7 Upscaling of log-normal permeability. The figures show the accuracy of the multiscale solution obtained after one iteration. In $\mathbf{a}$, we plot the $L^{\infty}$-error of the potential, and $\mathbf{b}$ shows neglected in the tangential component approximation. For problems involving irregular grids, MCDD-RBC and MCDD-TC do not preserve constant solution (see Section 3.6). By placing a high permeable channel between two opposite corners of the global fine grid, the tangential approximation (MCDD-TC and MCDD$\mathrm{RBC}$ ) is not able to capture the diagonal flow over the vertex nodes and requires solving many more finescale problems (iterations), even for Cartesian grid. The interface probing approximation is based on an algebraic approximation to the Schur complement and is more or less independent upon the geometry and principle directions on the fine scale. In Fig. 7a, b, we again observe that the MCDD-N preconditioner in general provides more accurate approximations to the solution after one iteration. The results in Fig. $7 b$ even show that the interface probing technique, using oscillating probing vectors, results in more accurate approximations to the multiscale flux on the fine scale than the more commonly used tangential component approximation. As for regular upscaling techniques, the quality of the solution-based probing vectors depends on the induced local boundary conditions and the size of the overlapping domains.

\subsection{Realistic porous media}

In realistic porous media, we might be faced with complex geological layers, where the fine-scale permeability has long and anisotropic correlation lengths.

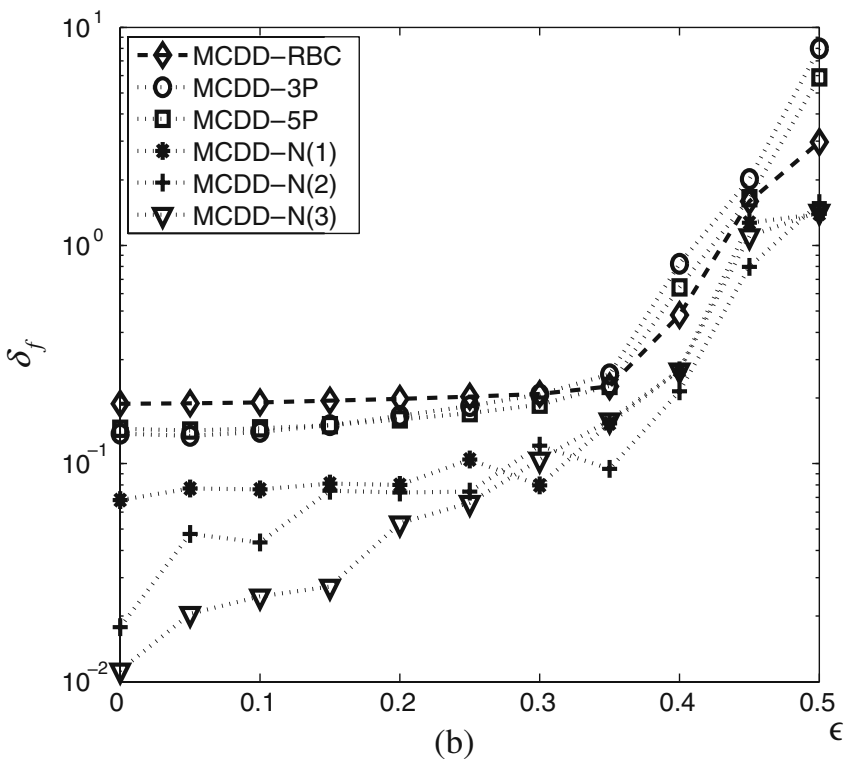

the $L^{\infty}$-error of the flux. All results are the truncated $(80 \%)$ mean values from 20 realisations of permeability fields and 20 realisations of randomly generated rough grids 
We consider the two-dimensional cross sections corresponding to the top and bottom layer of the 10th SPE comparative solution project [8] (see Fig. 8a, b). This test case is aimed at comparing different upscaling procedures and has been extensively used for testing multiscale methods. While the geometry is a simple uniform Cartesian grid, the permeability contrasts are rather challenging for reservoir simulators. The model for each layer consists of a $60 \times 220$ uniform Cartesian fine grid. To test the multiscale preconditioners, we further apply a $12 \times 20$ coarse grid. We use similar boundary conditions as in the previous test cases. The top layer is a Tarbert formation, while the bottom layer is fluvial. From the bottom layer (Fig. 8b), we clearly see channels with long correlation lengths throughout the reservoir. These channelized flow paths will result in anisotropic permeability on the coarse scale.

In Table 2, we compare the efficiency of the preconditioners based on the tangential flow approximation (MCDD-RBC) and the interface probing approximation (MCDD-3P and MCDD-5P). Since the simulation grid is $\mathbf{K}$-orthogonal, the MCDD-TC and MCDD-RBC are equivalent formulations. Thus, we only report the results of the MCDD-RBC. For the top layer (Tarbert formation; see Fig. 8a), there are quite large correlation lengths in the fine-scale permeability, with small subscale variations on each coarse block. All the preconditioners give quite good results for this problem. Note that even though the construction of the MCDD-3P and MCDD-5P requires solving 3 and 5 local fine-

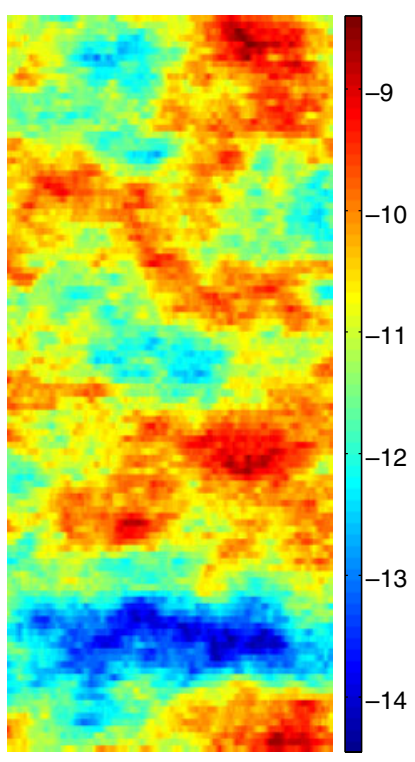

(a)

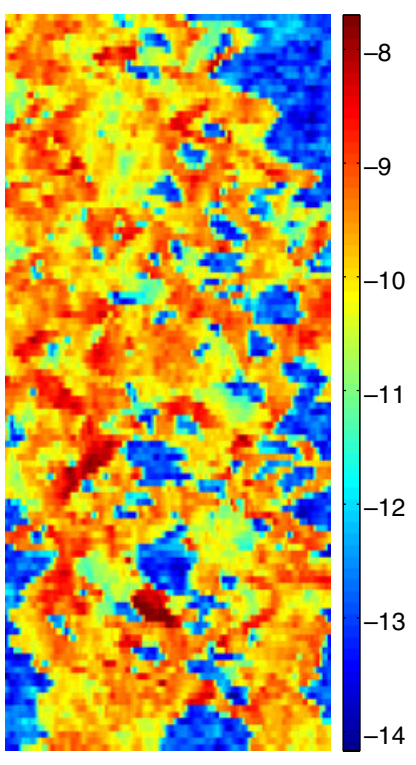

(b)
Fig. 8 Base 10 logarithm of permeability for the bottom and top layer of the SPE 10th comparative solution project. Here, a shows layer 1 (top) and b layer 85 (bottom)
Table 2 Number of fine-scale solves needed to reach a tolerance $10^{-8}$ in the $L^{2}$-norm

\begin{tabular}{lccc}
\hline Layer & MCDD-RBC & MCDD-3P & MCDD-5P \\
\hline 1 & 33 & 30 & 30 \\
85 & 347 & 167 & 146 \\
\hline
\end{tabular}

scale problems initially, they are still more efficient for solving the fine-scale problem iteratively. The bottom layer contains a fine-scale permeability with anisotropic correlation lengths. This channelized reservoir does not have a clear scale separation, which makes it more challenging to construct efficient multilevel preconditioners. Our results clearly show that the oscillating vectors of the interface probing technique (MCDD3P and MCDD-5P) are better suited for capturing the high-frequency error in the solution for channelized flow.

Table 3 shows the accuracy of the multiscale solution (first iteration). The results from layer 1 (top layer) show that we can construct quite accurate multiscale approximations to the fine-scale solution after only one iteration. Thus, there exists an exact representation of the flow on the coarse scale. By increasing the region of the overlap, the solution within the target region is less effected by the boundary conditions and more determined by the local variations in the fine-scale permeability. Thus, different boundary value problems will give more similar results, and the solution-based probing vectors can get close to linearly dependent. This may result in an illconditioned system for computing the interface approximation of MCDD-N, in which case we will have to reduce the number of solution-based probing vectors. A natural extension, which is beyond the scope of this paper, is to adapt the overlapping regions based on the fine-scale residual. This idea has been studied in [26].

In the results for layer 85 (bottom layer), the anisotropies in the upscaled permeability produce nonphysical oscillations in the solution for the coarse scale. In fact, the coarse-scale operators, for all the tested multiscale methods, fail to meet the requirements for monotonicity [28]. For completeness, we also tested the multiscale control volume method with linear boundary conditions as well as the MCDD-N preconditioner including global information. The MSFV method using linear boundary conditions has shown to give smaller errors then the MSFV method using reduced boundary conditions, for some problems involving highly anisotropic porous media [23]. By including global information in the MCDD-N preconditioners, the local approximations $\hat{S}_{E B}$ exactly reproduces the fine-scale flow field. However, these preconditioners also yield 
Table 3 The error of the multiscale solution after one iteration, measured in the $L^{\infty}$-norm

\begin{tabular}{lllllll}
\hline Layer & MCDD-RBC & MCDD-3P & MCDD-5P & MCDD-N(1) & MCDD-N(2) & MCDD-N(3) \\
\hline $1(\mathrm{u})$ & 0.15 & 0.14 & 0.19 & 0.032 & 0.043 & 0.018 \\
$1(\mathrm{f})$ & 0.069 & 0.098 & 0.12 & 0.012 & 0.014 & 0.037 \\
$85(\mathrm{u})$ & 0.47 & 11 & 155 & 2.6 & 0.54 & 0.53 \\
$85(\mathrm{f})$ & 0.43 & 0.58 & 8.1 & 2.8 & 0.26 & 0.17 \\
\hline
\end{tabular}

Here, $(u)$ denotes the fine-scale potential solution and $(f)$ the fine-scale flux

non-monotone coarse-scale operators and non-physical oscillations in the coarse-scale potential solution. Table 3 shows the large errors in the fine-scale potential solution, caused by non-physical oscillations on the coarse scale. These oscillations in turn result in wrong boundary conditions for the recalculation of fine-scale fluxes. As it is known that there exist cases where no nine-point scheme is monotone [21], changing the discretisation scheme might not be enough. In order to construct accurate upscaling methods for problems with anisotropic coarse-scale permeability, one approach might be to adapt the coarse-scale grid to the principal directions of the flow on the coarse scale. However, it is not clear how to construct a coarse grid which will guarantee monotonicity. In any case, we would need a robust multiscale framework which can handle simulations on general grids.

\section{Conclusion}

We have tested the efficiency and accuracy of MCDD preconditioners for two-dimensional heterogeneous elliptic problems on irregular grids. In the case of solving only one iteration, the MCDD preconditioners are similar to standard multiscale control volume methods where only the global coarse-scale equation is solved. Since we approximate the Schur complement, rather than the local basis functions, the proposed multiscale methodology is convergent to the fine scale. Our numerical experiments have shown that the reduced boundary condition (MCDD-RBC), commonly applied for constructing multiscale methods, is not robust with respect to perturbations on the fine scale. In the case of non-K-orthogonal grids, the reduced boundary condition does not preserve constant solutions, which makes it unsuitable for constructing multiscale preconditioners for flow problems on irregular grid structures or anisotropic permeability.

We have presented a more robust multiscale framework, based on the interface probing technique, for solving heterogeneous elliptic problems on irregular grids. While the MCDD-3P and MCDD-5P, using oscillating probing vectors, act as more efficient multiscale preconditioners for the fine-scale problem, solutionbased probing vectors can be constructed (MCDD-N) to give a more accurate representation of the coarse scale. Both of these preconditioners are purely algebraic upscaling techniques; thus, they are independent upon geometry and extendible to multiscale simulations on unstructured grids. Moreover, the interface probing technique can be seen as a more consistent way of incorporating global fine-scale information into the coarse-scale basis functions, as it is designed to exactly reproduce the solution of given boundary value problems.

When applied as a preconditioner for Krylov-type algorithms, the objective is to efficiently reduce the residual. At first iteration, the residual vector is exactly equal to the boundary conditions provided by the righthand side vector $p$. Thus, multiscale methods based on global information are accurate. After the first iteration, the residual is proportional to the error and is similar to oscillating noise. Thus, a simple fine-scale preconditioner, which effectively smooths the error, is to be preferred.

For certain anisotropy relations on the coarse scale, it is not possible to construct monotone nine-point stencils on the coarse scale. Thus, we may need to iterate on the fine-scale residual, in order to reduce the non-physical oscillations produced by the coarse scale. The MCDD preconditioners can be adapted to serve as either an accurate approximation to the coarse-scale problem (multiscale method) or an efficient preconditioner for the fine-scale problem.

Open Access This article is distributed under the terms of the Creative Commons Attribution Noncommercial License which permits any noncommercial use, distribution, and reproduction in any medium, provided the original author(s) and source are credited.

\section{References}

1. Aarnes, J.E.: On the use of a mixed multiscale finite element method for greater flexibility and increased speed or improved accuracy in reservoir simulation. Multiscale Model. Simul. 2(3), 421-439 (electronic) (2004) 
2. Aavatsmark, I.: An introduction to multipoint flux approximations for quadrilateral grids. Comput. Geosci. 6(3-4), 405432 (2002). Locally conservative numerical methods for flow in porous media

3. Arbogast, T.: Implementation of a locally conservative numerical subgrid upscaling scheme for two-phase Darcy flow. Comput. Geosci. 6(3-4), 453-481 (2002). Locally conservative numerical methods for flow in porous media

4. Axelsson, O.: Iterative Solution Methods. Cambridge University Press, New York (1994)

5. Bunch, J.R.: Stability of methods for solving Toeplitz systems of equations. SIAM J. Sci. Statist. Comput. 6(2), 349-364 (1985)

6. Chan, T.F.: Analysis of preconditioners for domain decomposition. SIAM J. Numer. Anal. 24(2), 382-390 (1987)

7. Chan, T.F.C., Mathew, T.P.: The interface probing technique in domain decomposition. SIAM J. Matrix Anal. Appl. 13(1), 212-238 (1992)

8. Christie, M., Blunt, M.: Tenth SPE comparative solution project: A comparison of upscaling techniques. SPE Reserv. Evalu. Eng. 4(4), 308-317 (2001)

9. Chu, J., Efendiev, Y., Ginting, V., Hou, T.Y.: Flow based oversampling technique for multiscale finite element methods. Adv. Water Resour. 31(4), 599-608 (2008)

10. Durlofsky, L., Efendiev, Y., Ginting, V.: An adaptive localglobal multiscale finite volume element method for twophase flow simulations. Adv. Water Resour. 30(3), 576-588 (2007)

11. Efendiev, Y., Ginting, V., Hou, T., Ewing, R.: Accurate multiscale finite element methods for two-phase flow simulations. J. Comput. Phys. 220(1), 155-174 (2006)

12. Farmer, C.L.: Upscaling: a review. Int. J. Numer. Methods Fluids 40(1-2), 63-78 (2002). ICFD Conference on Numerical Methods for Fluid Dynamics (Oxford, 2001)

13. Ginting, V.: Analysis of two-scale finite volume element method for elliptic problem. J. Numer. Math. 12(2), 119-141 (2004)

14. Golub, G.H., Mayers, D.: The use of preconditioning over irregular regions. In: Computing Methods in Applied Sciences and Engineering, VI (Versailles, 1983), pp. 3-14. NorthHolland, Amsterdam (1984)

15. Graham, I.G., Lechner, P.O., Scheichl, R.: Domain decomposition for multiscale PDEs. Numer. Math. 106(4), 589-626 (2007)

16. Hajibeygi, H., Bonfigli, G., Hesse, M.A., Jenny, P.: Iterative multiscale finite-volume method. J. Comput. Phys. 227(19), 8604-8621 (2008)
17. Hesse, M.A., Mallison, B.T., Tchelepi, H.A.: Compact multiscale finite volume method for heterogeneous anisotropic elliptic equations. Multiscale Model. Simul. 7(2), 934-962 (2008)

18. Hou, T.Y., Wu, X.H.: A multiscale finite element method for elliptic problems in composite materials and porous media. J. Comput. Phys. 134(1), 169-189 (1997)

19. Hou, T.Y., Wu, X.H., Cai, Z.: Convergence of a multiscale finite element method for elliptic problems with rapidly oscillating coefficients. Math. Comput. 68(227), 913-943 (1999)

20. Jenny, P., Lee, S.H., Tchelepi, H.A.: Multi-scale finitevolume method for elliptic problems in subsurface flow simulation. J. Comput. Phys. 187(1), 47-67 (2003)

21. Keilegavlen, E., Nordbotten, J.M., Aavatsmark, I.: Sufficient criteria are necessary for monotone control volume methods. Appl. Math. Lett. 22(8), 1178-1180 (2009)

22. Kippe, V., Aarnes, J.E., Lie, K.A.: A comparison of multiscale methods for elliptic problems in porous media flow. Comput. Geosci. 12(3), 377-398 (2008)

23. Lunati, I., Jenny, P.: Treating highly anisotropic subsurface flow with the multiscale finite-volume method. Multiscale Model. Simul. 6(1), 308-318 (electronic) (2007)

24. Mandel, J.: Balancing domain decomposition. Commun. Numer. Methods Eng. 9(3), 233-241 (1993)

25. Mandel, J., Sousedík, B.: Coarse spaces over the ages Arxiv e-prints (2009)

26. Nordbotten, J.M.: Adaptive variational multiscale methods for multiphase flow in porous media. Multiscale Model. Simul. 7(3), 1455-1473 (2008)

27. Nordbotten, J.M., Aavatsmark, I., Eigestad, G.T.: Monotonicity of control volume methods. Numer. Math. 106(2), 255288 (2007)

28. Nordbotten, J.M., Bjørstad, P.E.: On the relationship between the multiscale finite-volume method and domain decomposition preconditioners. Comput. Geosci. 12(3), 367-376 (2008)

29. Saad, Y., Schultz, M.H.: GMRES: a generalized minimal residual algorithm for solving nonsymmetric linear systems. SIAM J. Sci. Statist. Comput. 7(3), 856-869 (1986)

30. Sandvin, A., Nordbotten, J.M., Aavatsmark, I.: A unified framework of upscaling and domain decomposition. In: XVIII Conference on Computational Methods in Water Resources (2010)

31. Smith, B.F., Bjørstad, P.E., Gropp, W.D.: Domain Decomposition. Cambridge University Press, Cambridge (1996). Parallel multilevel methods for elliptic partial differential equations 